Article original

\title{
Prise en charge des patients nécessitant une réintervention cardiaque valvulaire : Expérience du service de cardiologie du CHU d'Oran
}

\author{
Management of patients requiring a reintervention of the heart valve: Expe- \\ rience of the cardiology department of University Hospital Center of Oran
}

Nadia LAREDJ, Farouk BOUKERCHE, Lilia ZOULI, Leila HAMMOU

Service de Cardiologie, CHU Oran. Faculté de Médecine d'Oran

Auteur correspondant: Idj_nadia@yahoo.fr

Article reçu le 17/03/2019 Accepté le 26/06/2019

\section{MOTS CLÉS \\ Réintervention valvu- laire, dysfonction de prothèse, endocardite, valvulopathie négligée}

\section{Résumé}

Introduction - Les cardiopathies valvulaires demeurent fréquentes malgré l'amélioration des conditions sanitaires qui ont permis la réduction de l'incidence du rhumatisme articulaire aigu (RAA). Les indications chirurgicales et interventionnelles se sont élargies et nous opérons aujourd'hui à des stades précoces voire asymptomatiques. Une réintervention chirurgicale valvulaire est nécessaire dans environ $15 \%$ des cas au cours de l'évolution d'une valvulopathie opérée. L'objectif de notre étude est de déterminer la fréquence des réinterventions valvulaires et d'identifier les principales indications de ces réinterventions.

Méthodes - Il s'agit d'une étude rétrospective descriptive, portant sur 45 patients, admis au service de cardiologie du Centre Hospitalo-Universitaire (CHU) d'Oran, de janvier 2011 à juillet 2013, et présentant une valvulopathie déjà opérée nécessitant une autre réintervention chirurgicale valvulaire.

Résultats - La moyenne d'âge est de 41 ans, $73 \%$ des patients sont des femmes et $27 \%$ des hommes (Sex ratio de 0,37). La plupart de nos patients sont symptomatiques (dyspnée stade II de la NYHA). Les réinterventions intéressent la valve mitrale dans $69 \%$ des cas, la valve aortique dans $37 \%$ des cas et la valve tricuspide dans $35 \%$. Nous avons constaté l'importance des insuffisances aortiques et tricuspides négligées lors de la première intervention (31\% et $62 \%$ respectivement). Le délai moyen entre les deux interventions, tout type confondu, est de 21 ans.

Conclusion - Les réinterventions cardiaques peuvent survenir au cours de l'évolution d'une valvulopathie. Une bonne évaluation cardiaque initiale des valvulopathies lors d'une chirurgie portant sur la valve mitrale ou aortique est indispensable pour pallier au problème d'une réintervention pour des valvulopathies négligées. 


\section{KEY WORDS}

valvular reintervention, prosthesis dysfunction, endocarditis, neglected valvular disease

\section{Introduction}

Les réinterventions valvulaires occupent une place importante dans la chirurgie cardiaque. Il s'agit d'un réel problème de santé publique. Une réintervention valvulaire est nécessaire dans environ $15 \%$ des cas au cours de l'évolution d'une valvulopathie [1]. Les causes de ces réinterventions sont diverses et multiples : endocardite infectieuse, dysfonction de prothèse, détérioration de la plastie, apparition d'une autre valvulopathie, etc...

Ces réinterventions s'accompagnent d'une surmortalité variable en fonction des patients et surtout du type de l'intervention [1,2]. En effet, Le risque d'une réopération augmente après chaque intervention, et diffère pour les prothèses mécaniques et biologiques, ainsi que pour les plasties [2]. Il est plus élevé en position mitrale, qu'en position aortique [2]. Il est important de ce fait d'étudier les causes des réinterventions en termes de santé publique afin de réduire leur incidence. Les objectifs de notre étude sont de déterminer la fréquence des réinterventions valvulaires ainsi que leurs principales indications.

\section{Matériel et méthodes}

Il s'agit d'une étude descriptive rétrospective portant sur 45 patients admis au service de cardiologie du Centre Hospitalo-Universitaire (CHU) d’Oran, de janvier 2011 à juillet 2013, et présentant une valvulopathie déjà opérée nécessitant une réintervention chirurgicale valvulaire.

On a noté la présentation clinique et la symptomatologie du patient, les données de l'examen clinique, la date de la première intervention ainsi que le type d'intervention (geste conservateur ou remplacement valvulaire, prothèse mécanique ou biologique), et le délai entre les 2 interventions.

Nous avons collecté également les données de l'électrocardiogramme et de l'échocardiographie transthoracique voire transoesophagienne: l'atteinte valvulaire (la quantifier et apprécier sa sévérité), l'existence ou non de dysfonction de prothèse, de lésions d'endocardite, la mesure de la fraction d'éjection ventriculaire gauche FEVG, la mesure des pressions pulmonaires et l'étude de la fonction ventriculaire droite. Et enfin, il est précisé le traitement du patient et l'indication opératoire retenue par le staff médico-chirurgical. 
Les données ont été répertoriées sur un fichier EXCEL avec calcul des moyennes et des pourcentages.

\section{Résultats}

La moyenne d'âge est de 41 ans, $73 \%$ des patients sont des femmes et $27 \%$ des hommes (sex ratio égal à 0,37 ). Les valvulopathies sont toutes d'étiologie rhumatismale. Dans $89 \%$ des cas, les premières interventions ont concerné un geste sur la valve mitrale, $22 \%$ sur la valve aortique, tandis que la valve tricuspide était moins fréquemment atteinte: $16 \%$ des cas, rarement la valve pulmonaire avec un taux de $2 \%$.

Sur le plan fonctionnel, $95 \%$ des patients sont symptomatiques, présentant au moins une dyspnée stade II de la NYHA. Un tiers des patients avaient une fraction d'éjection ventriculaire gauche diminuée ( $\mathrm{FEVG}<50 \%$ ) et $64 \%$ des patients étaient en fibrillation auriculaire.

Toutefois, les réinterventions intéressent la valve mitrale dans $69 \%$ des cas, la valve aortique dans $37 \%$ des cas et la valve tricuspide dans $35 \%$ (figure 1 ).

Figure 1. Répartition des réinterventions selon la valve atteinte

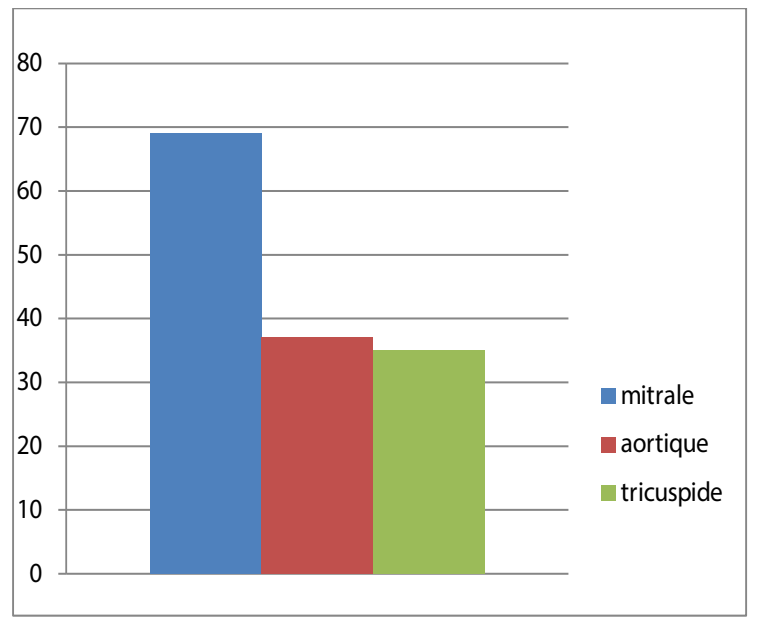

Concernant la valve mitrale, les réinterventions sont motivées dans la plupart des cas par une resténose après commissurotomie à cœur ouvert (CCO) (84\%), et dans $7 \%$ des cas par une dysfonction de prothèse, dans $6 \%$ des cas par une plastie sténosante ou fuyante, et dans $3 \%$ des cas pour une valvulopathie mitrale apparue après une première intervention touchant une autre valve (insuffisance mitrale significative ou rétrécissement mitral devenant serré) (figure 2).
Figure 2. Les différentes causes de réintervention pour la valve mitrale

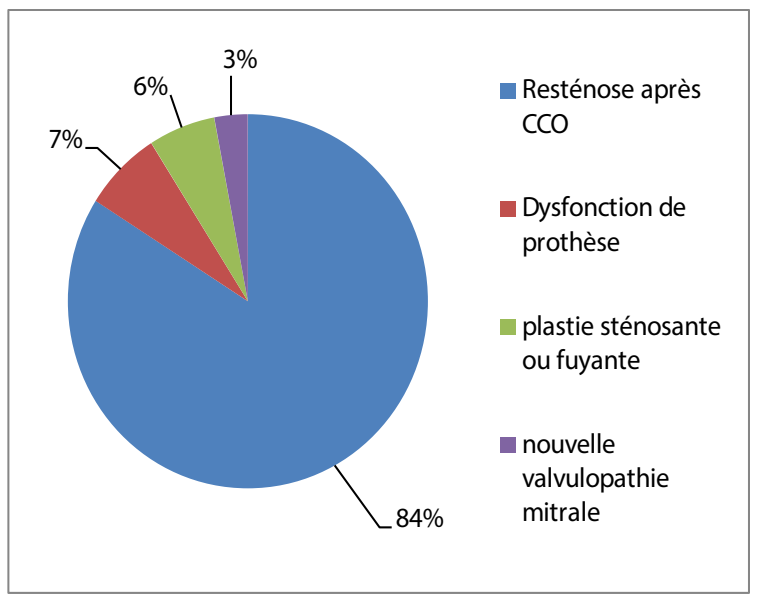

Pour les réinterventions sur la valve aortique, elles sont indiquées dans $44 \%$ des cas pour une dysfonction de prothèse aortique, alors que $56 \%$ des cas sont motivés par une nouvelle valvulopathie aortique avec l'importance du nombre des insuffisances aortiques négligées dans 31\% des cas.

La réintervention sur la valve tricuspide est indiquée dans $62 \%$ des cas pour une nouvelle fuite tricuspide, et dans $38 \%$ des cas pour une plastie tricuspide fuyante ou sténosante.

Nous avons observé que la reprise chirurgicale pour une autre valvulopathie représentait $11 \%$ des cas avec une prédominance des fuites aortiques.

Le délai moyen entre les deux interventions, tout type confondu, est de 21 ans, mais celui- ci est variable si nous prenons en considération la technique de réparation initiale.

Le geste effectué lors de la seconde intervention est le remplacement valvulaire dans $80 \%$ des cas.

\section{Discussion}

Dans notre série, les principales indications de réintervention ont intéressé la valve mitrale, représentées essentiellement par la resténose mitrale après commissurotomie à cœur ouvert, suivies par les dysfonctions de prothèses mécaniques, mitrales et aortiques, et enfin les fuites valvulaires, aortiques et tricuspides, négligées lors de la première intervention, devenant significatives et gênantes pour les patients. Les patients de notre série étaient symptomatiques ce qui a motivé les indications de réinterventions et ont nécessité dans $80 \%$ des cas un remplacement valvulaire par une prothèse mécanique lors de la réintervention. 
Un malade subissant une chirurgie cardiaque, vivant de plus en plus longtemps, la réintervention devient quasi inéluctable et fait partie de l'histoire naturelle du patient qui doit être prévenu de cette éventualité [1]. Parmi les porteurs de valves mécaniques, $2 \%$ ont besoin de réintervention chaque année avec un pic de fréquence vers le 3ème mois en relation avec un plus grand risque d'endocardite sur prothèse $[1,2]$. Cette fréquence diminue progressivement avec le temps [2]. Le taux de survie à 5 ans, sans réopération est de 96 à $99 \%$ avec les valves à doubles ailettes actuellement utilisées. Ce risque est d'autant plus élevé avec les anciennes prothèses à billes $(0,7$ à $1,1 \%$ par an) $[3,4]$.

Concernant les bioprothèses, elles présentent deux pics de réopération, un premier se situant vers les trois premiers mois, un second tardif lié à la dégénérescence de la valve $[4,5]$. La survie sans opération pour ces valves passe de $97 \%$ à 5 ans à $55 \%$ à 15 ans $[4,5]$. Toutefois, cette détérioration varie selon la tranche d'âge des patients : elle est la plus élevée chez les sujets jeunes et diminue de façon significative chez les plus de 70 ans $[4,5]$.

La mortalité des réinterventions est supérieure à celle d'un premier remplacement valvulaire [6]. Elle est très variable et se situe entre 3 et $13 \%[2,6]$. La survie diminue ainsi en cas de réinterventions itératives à $52 \%$ à 5 ans et $33 \%$ à 10 ans [2].

Il est important de noter que le dysfonctionnement du ventricule gauche et la classe IV de la New York Health Asoociation (NYHA) constituent des facteurs prédictifs indépendants de mortalité hospitalière et à long terme [2,7]. Les autres facteurs de risque de mortalité et de morbidité lors des réinterventions valvulaires sont la présence d'une insuffisance tricuspidienne sévère, l'intervention en urgence, le nombre de réinterventions, l'endocardite, les besoins d'un geste concomitant tel un pontage aorto-coronarien et l'insuffisance rénale [7]. D'autres facteurs per opératoires augmentent le risque chirurgical, tels que la durée du clampage aortique ou la durée de la circulation extracorporelle. L'âge avancé, plus de 70 ans, est lié à une mortalité plus élevée avec $32 \%$ versus $17 \%$ pour les moins de 70 ans, et $4 \%$ pour les patients âgés de moins de 60 ans [7]. La morbidité est en moitié liée au saignement per opératoire et aux transfusions multiples qui en résultent, ce saignement est en rapport avec la dissection des adhérences et la durée prolongée de la circulation extracorporelle $[1,2,7]$.

Dans notre série, nous avons constaté que le délai moyen entre la première et la seconde intervention est en moyenne de 21 ans, lié d'une part au retard que mettent les patients à consulter et d'autre part à la disponibilité d'un centre chirurgical.
Plus de $70 \%$ des réinterventions dans notre série intéressent la valve mitrale et sont en majorité en rapport avec des resténoses post dilatation. Le rhumatisme articulaire aigu à l'origine de ces valvulopathies et qui reste prépondérant dans la population Algérienne, explique ces résultats. De plus, les commissurotomies percutanées (CMP) ou chirurgicales étaient réservées à des femmes en âge de procréation, dans l'idée de les réopérer à un âge plus avancé. Les principaux facteurs de risque de mauvais résultat immédiat d'une commissurotomie percutanée (CMP) sont une anatomie valvulaire mitrale défavorable mais aussi un âge avancé et une sténose mitrale très serrée (surface mitrale $<1 \mathrm{~cm}^{2}$ ) [8]. Dans la série de lung et al [8], qui a inclut 1514 patients, moyenne d'âge 45 ans, où un modèle logistique a identifié les facteurs prédictifs de bons résultats immédiats de la CMP qui sont l'âge $(p=0,004)$ et une surface mitrale $\geq 1,5 \mathrm{~cm}^{2}$ $(p<0,0001)$ L'analyse des résultats tardifs, jusqu'à 15 ans, confirme l'efficacité de la méthode [9]. La qualité du résultat à distance dépend étroitement du résultat immédiat.

En cas de mauvais résultat immédiat lié à une insuffisance mitrale importante ou à une ouverture mitrale insuffisante, l'évolution est rapidement défavorable et la chirurgie est nécessaire à court terme. Inversement, après une commissurotomie percutanée (CMP) réussie, une amélioration fonctionnelle est obtenue dans $60 \%$ des cas à 10 ans [10]. Ces résultats soulignent l'importance de prendre en compte toutes les caractéristiques cliniques et surtout échocardiographiques pour la sélection des patients avant commissurotomie, quelle soit percutanée ou chirurgicale [11], afin de retarder une éventuelle réintervention à long terme. Nos patients ont été réopérés en moyenne 10 ans après commissurotomie car l'anatomie valvulaire initiale était favorable et la sélection des patients était optimale.

Dans notre étude, la quasi-totalité des réinterventions sur prothèse aortique sont en rapport avec une dysfonction de cette prothèse : mismatch, pannus fibreux, endocardite, et surtout thrombose de prothèse. L'insuffisance du traitement anticoagulant est fréquente chez nos patients, malgré les efforts d'éducation entrepris, favorisée par une mauvaise observance du traitement, les changements d'alimentation, les interactions médicamenteuses, et les bilans d'hémostase non réalisés par le patient. Cependant, dans $31 \%$ des cas les réinterventions intéressent des insuffisances aortiques négligées, chez des patients porteurs de prothèse mitrale, d'où l'importance d'une évaluation cardiaque précise en cas de polyvalvulopathie, qui sont toujours aussi fréquentes dans la population Algérienne, apanage du RAA. Elles tirent leurs particularités de la grande variabilité des tableaux rencontrés, en raison de la diversité des associations avec, à des degrés divers, fuite et sténose sur chacun des orifices valvulaires [11]. L'échocardiographie transthoracique permet un bilan lésionnel complet mais il faut souligner l'intérêt de 
l'échocardiographie trans-oesophagienne (ETO) qui permet d'affiner l'analyse des différentes valves et de quantifier l'importante des régurgitations, en particulier les fuites aortiques qui paraissent négligeables en échographie transthoracique alors que réellement elles ne le sont pas [12-14].

Il est important également de signaler les reprises chirurgicales pour des insuffisances tricuspides négligées ou mal évaluées lors de la première intervention. Dans notre série, la réparation de la valve tricuspide est indiquée dans $62 \%$ des cas pour une nouvelle fuite tricuspide probablement négligée lors de la première intervention, et dans $38 \%$ des cas la plastie tricuspide était fuyante ou sténosante. Il faut rappeler que le traitement des insuffisances tricuspides fonctionnelles volumineuses est avant tout préventif et repose sur la nécessité d'un geste chirurgical mitral et/ ou aortique précoce, avant l'apparition de la dilatation de l'anneau tricuspide et/ou de la dysfonction ventriculaire droite [12-23]. Les résultats de la chirurgie tricuspide isolée et tardive apparaissent médiocres, avec une importante mortalité périopératoire comprise entre $9 \%$ et $50 \%$ et cette dernière concerne le remplacement valvulaire tricuspide par bioprothèse exclusivement [24,25]. La morbi-mortalité tardive après une telle réintervention apparait également très importante, puisque seulement $42 \%$ des patients sont indemnes d'évènements cardiaques (décès ou réinterventions) à 5 ans selon Staab et al [24]. Cette série [24] avait inclus 34 patients qui présentaient une insuffisance tricuspide sévère et symptomatique, avec une mortalité hospitalière précoce de $8.8 \%$. Des études récentes encourageraient la correction de cette insuffisance tricuspide par voie percutanée, en particulier chez les patients inopérables [26-31].

Pour pallier au problème des insuffisances tricuspides négligées, l'annuloplastie tricuspide devient alors systématique, associée au geste mitral, lorsque l'anneau tricuspide est dilaté de manière isolée, même en l'absence d'insuffisance tricuspide importante ou lorsque le diamètre échographique de l'anneau tricuspide est supérieur à $21 \mathrm{~mm} / \mathrm{m}^{2}$ de surface corporelle. Dans ces cas et au moindre doute, il ne faut pas hésiter à réaliser une échographie trans-oesophagienne pour mieux évaluer l'anneau tricuspide [11-14].

\section{Conclusion}

Les réinterventions cardiaques peuvent survenir au cours de l'évolution d'une valvulopathie. Pour cela, le patient doit être impérativement prévenu de cette éventualité et la possibilité d'une réintervention devra être envisagée dès la première chirurgie cardiaque, en particulier en cas de resténose mitrale après commissurotomie percutanée ou chirurgicale comme le montre notre série ou en cas de dysfonction de prothèse mécanique. Le moment de la réintervention ne doit pas être retardé, car les co-morbidités cardiaques ou extracardiaques majorent le risque d'une deuxième intervention. L'avènement des techniques percutanées a largement amélioré la survie de ces patients. Enfin, une bonne évaluation cardiaque initiale des autres valvulopathies, lors d'une chirurgie portant sur la valve mitrale ou aortique, est indispensable pour pallier au problème d'une réintervention pour des valvulopathies aortiques ou tricuspides négligées.

\section{Conflits d'intérêt}

Les auteurs déclarent n'avoir aucun conflit d'intérêt.

\section{Références bibliographiques}

1.Jebara VA et Achouh PE. Réinterventions valvulaires pour valvulopathies acquises. Cardiopathies valvulaires acquises. Jean et christophe ACAR. Médecine-sciences Flammarion 2000. Chapitre 36 : pages 501-506.

2.Caus T, Mesana T, Mouly A et al. Remplacements valvulaires itératifs : Pronostic et résultats. Arch Mal Cœur, 1995, 88 :35-41.

3.Acar J. la chirurgie cardiaque valvulaire : un demi-siècle d'histoire. Arch Mal Cœur Vaiss, 1995,88 :1327-1333.

4.Jamieson WRE. Mechanical and bioprosthetic aortic valve replacement: LH Edmunds. Cardiac surgery in the adult, New York, McGraw-Hill, 1997:859-910.

5.Akins C, Buckley M, Daggett W et al. risk of reoperative valve replacement for failed mitral and aortic bioprotheses. Ann Thorac Surg 1998. 65:1545-1552.

6. Husebeye DG, Pluth JR, Piehler JM et al. Reoperation on prothetic heart valves. An analysis of risk factors in 552. J Thoracic Cardiovasc Surg 1983. 1983,86:534-552.

7.Cohen L, Aranki S, Rizzo R et al. Decrease in operative risk of reoperative valve surgery. Ann Thoracic Surg. 1993, 56:15-21.

8. Lung B, Cormier B, Ducimetière $P$ et al. Immediate results of percutaneous mitral commissurotomy : a predictive model on a series of 1514 patients. Circulation 1996; 94:2124-30.

9.Palacios I, Sanchez P, Harell L et al. Which patients benefit from percutaneous mitral balloon valvuloplasty : Prevalvuloplasty ans post valvuloplasty variables that predict long term outcome. Circulation 2002; 105:1465-71.

10.Lung B, Garbarz E, Michaud P et al. Late results of percutaneous mitral commissurotomy in a series of 1024 patients. Circulation 1999; 99:3272-8. 
11. Tribouilloy C, Acar C, Cormier B et al. Recommandation des la société française de cardiologie concernant la prise en charge des valvulopathies acquises et des dysfonctions de prothèses valvulaires. Arch Mal Cœur Vaiss 2005, tome 98 ; N 2 (suppl) : p5-61.

12.Bonow et al. ACC/AHA guidelines for the management of patients with valvular Heart disease. JACC 2006, vol 48, $\mathrm{N}^{\circ}$ 3: e1-148.

13.G de Gevigney. A propos des nouvelles recommandations européennes sur la prise en charge des valvulopathies. Arch Mal Cœur Vaiss 2007. Tome 100, $\mathrm{N}^{\circ} 9: 775-781$.

14. The Task Force for the Management of Valvular Heart Disease of the European Society of Cardiology (ESC) and the European Association for Cardio-Thoracic Surgery (EACTS). European Heart Journal, Volume 38, Issue 36, 21 September 2017, Pages 2739-2791,

15. Sugimoto D, Okada M, Yamashita $C$ et al. Surgical assessment of tricuspid valve replacement for severe tricuspid regurgitation without stenosis. Ann Thorac Cardiovasc Surg 1999, 5:300-303.

16. Matsuyama K, Matsumoto M, sugita T et al. Predictors of residual tricuspid regurgitation after mitral valve surgery. Ann Thorac Surg 2003; 75:1826-8.

17.King R, Schaff H, Dianelson G, surgery for tricuspid regurgitation late after mitral valve replacement. Circulation 1984; 70(Suppl 1):937.

18.Cohn LH. Tricuspid regurgitation secondary to mitral valve disease: When and how to repair. J Card Surg 1994; 9:237-4.

19.Pellegrini A, Colombo T, Donatelli $F$ et al. Evaluation and treatment of secondary tricuspid insufficiency. Eur J Cardiothorac Surg 1992; 6:288-96.

20.Bianchi G, Solinas M, Bevilacqua S, Glauber M. Which patient undergoing mitral valve surgery should also have the tricuspid repair? Interact Cardiovasc Thorac Surg. 2009(6):1009-20.

21. Izumi C, Iga K, Konishi T. Progression of isolated tricuspid regurgitation late after mitral valve surgery for rheumatic mitral valve disease. J JHeart Valve Dis. 2002; 11(3): 353-6.

22. Shiran A, Sagie A. Tricuspid regurgitation in mitral valve disease incidence, prognostic implications, mechanism, and management. J Am Coll Cardiol. 2009; 53(5): 401-8.

23. Rogers JH, Bolling SF. The tricuspid valve: current perspective and evolving management of tricuspid regurgitation. Circulation. 2009 May 26; 119(20):2718-25.

24.Staab ME, Nishimura, Dearani JA. Isolated tricuspid valve surgery for severe tricuspide regurgitation following prior left heart valve surgery: analysis of outcome in 34 patients. J Heart Valv Dis 1999; 8:567-74.

25. Hornick P, Harris PA, Taylor KM. tricuspid valve replacement subsequent to previous open heart surgery. J Heart Valv Dis 1996; 5:20-5.

26.Rodés-Cabau J, Hahn RT, Latib A, Laule M, Lauten A, Maisano F, Schofer J, Campelo-Parada F, Puri R, Vahanian A. Transcatheter The- rapies for Treating Tricuspid Regurgitation. J Am Coll Cardiol. 2016 Apr 19; 67(15):1829-1845.

27.Figulla HR, Kiss K, Lauten A. Transcatheter interventions for tricuspid regurgitation - heterotopic technology: TricValve. Eurolntervention. 2016 Sep 18;12(Y): Y116-8.

28. Haude M, Prendergast B. Percutaneous interventional options for the treatment of tricuspid regurgitation. Eurointervention 2016 Sep 18;12(Y):Y107.

29. Taramasso M, Hahn RT, Alessandrini H, et al. The International Multicenter TriValve Registry: Which Patients are undergoing Transcatheter Tricuspid Repair? JACC Cardiovasc Interv. 2017 Oct 9;10(19):1982-1990.

30. Aboulhosn J, Cabalka AK, Levi DS, et al. Transcatheter Valve-inRing Implantation for the Treatment of Residual or Recurrent Tricuspid Valve Dysfunction After Prior Surgical Repair. JACC Cardiovasc Interv. 2017 Jan 9; 10(1):53-63.

31.Perlman G, Praz F, Puri R, Ofek H et al. Transcatheter Tricuspid Valve Repair With a New Transcatheter Coaptation System for the Treatment of Severe Tricuspid Regurgitation: 1-Year Clinical and Echocardiographic Results. JACC Cardiovasc Interv. 2017 Oct 9; 10(19):1994-2003. 